\title{
3 Research Square \\ Clinical Features of POEMS Syndrome In Southeast Asia: A Literature-Based Study
}

Mario B. Prado Jr ( $\square$ mbprado@alum.up.edu.ph )

Chiba University https://orcid.org/0000-0001-5756-7321

Karen Joy Adiao

University of the Philippines-Manila

\section{Research Article}

Keywords: POEMS Syndrome, Southeast Asia, Crow Fukase Syndrome, Prevalence, Clinical Features

Posted Date: December 29th, 2021

DOl: https://doi.org/10.21203/rs.3.rs-893599/v1

License: (9) This work is licensed under a Creative Commons Attribution 4.0 International License. Read Full License

Version of Record: A version of this preprint was published at SN Comprehensive Clinical Medicine on January 31st, 2022. See the published version at https://doi.org/10.1007/s42399-022-01130-3. 


\section{Abstract}

Purpose: To determine and analyze the clinical characteristics of POEMS Syndrome among Southeast Asian countries.

Methods: We searched the literature using a pre-specified inclusion and exclusion criteria and using the search terms "[(POEMS) or (Takatsuki) or (PEP) or (Crow Fukase) and (syndrome)] AND [Countries/People of Southeast Asia]".

Results: Seven studies, including 5 case reports, 1 case series and 1 correspondence letter containing 8 patients were eligible for analysis. The median age of onset was 54 years, while the median duration to correct diagnosis was 5.5 months. The most common initial presentation was weakness (4/6) with $50 \%$ initially diagnosed as chronic inflammatory demyelinating polyneuropathy. On physical examination, $100 \%$ had evidence of length dependent polyneuropathy, $80 \%$ had papilledema, $75 \%$ had edema/effusion, $86 \%$ had skin changes and $67 \%$ had organomegaly. All had abnormal NCS and CT scan while 1 tested negative for monoclonal gammopathy restricted to lambda. Only 2 had VEGF results, one of which was normal. Melphalan and steroid combination was the most common treatment given with only 1 case dying of sepsis.

Conclusion: Although the number of cases in Southeast Asia is lower, which can be attributed to difference in ethnicity and geographical location, the presenting signs and symptoms of this condition was similar to other countries. However, the new proposed criteria may not be applicable in the region as only few countries are capable of doing VEGF testing.

\section{Introduction}

POEMS Syndrome is a rare monoclonal gammopathy characterized predominantly by polyneuropathy, organomegaly, endocrinopathy, $M$-protein and skin changes. ${ }^{1,2}$ Not all conditions represented in the acronym are needed for its diagnosis as other manifestations such as Castleman disease, sclerotic bone lesions, papilledema, effusion or edema, polycythemia, erythrocytosis or thrombocytosis, mostly thought to be secondary to elevated vascular endothelial growth factor (VEGF) levels, may be required to satisfy the criteria for its occurrence. ${ }^{3}$ Despite the availability of several criteria, diagnosing POEMS syndrome is a challenge due to its multisystemic affection. ${ }^{1,4,5}$ Only around $60 \%$ will initially present with polyneuropathy and the same proportion of patients will be mislabeled as chronic inflammatory demyelinating polyneuropathy (CIDP) on first encounter. ${ }^{6,7}$ Early and correct diagnosis is of utmost importance as prognosis is poor. ${ }^{3}$ In a retrospective study conducted in Japan in the 1980 s, $66 \%$ of 58 patients died, with a mean survival time of 33 months. ${ }^{5}$ Nevertheless, in recent years, due to increased awareness and improvement in the battery of management options such as radiotherapy, autologous stem cell therapy with high dose chemotherapy, use of immunomodulators and protease inhibitors, long term survival has drastically improved. ${ }^{3,4,8}$ POEMS syndrome was coined by Bardwick in the 1980 s but 
was first documented by Crow in the 1950 s. ${ }^{9}$ It is also known as Takatsuki Syndrome, Crow-Fukase Syndrome or PEP Syndrome (Plasma Cell Dyscrasia, Endocrinopathy, Polyneuropathy). ${ }^{3}$

Despite its rarity, POEMS Syndrome is relatively more common in East Asian than Southeast Asian Countries. A national survey in Japan in 2019 revealed a prevalence of $0.3 / 100,000 .{ }^{10}$ Meanwhile, a recent literature review of all cases published in China identified a total of 1946 cases from 1986 to 2016. ${ }^{7}$ While case series were also reported in US $^{1}$ and India ${ }^{11}$, the true prevalence is still unknown. No case series of more than five patients were published in any of the 11 countries in Southeast Asia to date.

While there is no published evidence on the relationship of geography and ethnicity to POEMS syndrome, conditions like multiple sclerosis ${ }^{12}$ and X-linked dystonia parkinsonism (XDP) ${ }^{13}$ are known to distribute more in the northern hemisphere and only to Filipinos respectively, and may explain the disparity in prevalence. Besides this, clinical features and response to treatment also differ between countries and ethnicities. Whereas American patients respond readily to radiotherapy and Japanese to immunomodulators, the reverse can't be assumed otherwise. ${ }^{1,3}$ Likewise, prognosis is also affected by race. $^{7}$

Therefore, the main objective of this study is to search for published cases of POEMS syndrome in the 11 countries in Southeast Asia, namely: Singapore, Philippines, Malaysia, Indonesia, Thailand, Brunei, Vietnam, Cambodia, Laos, Myanmar and Papua New Guinea, and determine and analyze its clinical characteristics among Southeast Asian people.

\section{Materials And Methods}

\subsection{Search Process}

Two review authors (MBP and KJA), both neurologists conducted the search, and selected the reports. Whenever a disagreement was present, a third-party consultant (KP) made the decision. The following search terms were used in Pubmed Medline, Cochrane and Embase (see supplemental material):

\section{1. (POEMS OR TAKATSUKI OR CROW-FUKASE OR PEP) AND (Syndrome)}

2. Malay (Including all population in Southeast Asia)

\section{3. \#1 AND \#2}

The search strategy terms were formulated last December 2020 and approved by authors. No additional filters were included. Additional journals from references whenever applicable were also reviewed. Titles and abstracts of the initial results were screened for duplication and relevance. Full articles of the unrejected studies were collected and reviewed. Authors of studies were sent electronic mails to gather more information if needed. Ongoing and unpublished data sources were also searched whenever possible by using RIAT. Articles that satisfied the eligibility criteria were included in the analysis. 


\subsection{Eligibility Criteria}

Only studies involving people of Malay descent were included. Specifically, studies involving Singaporeans, Malaysians, Filipinos, Thais, Cambodians, Indonesians, Vietnamese, Lao and people from Myanmar and Papua New Guinea were analyzed. Cases should have satisfied POEMS Syndrome criteria by Dispenzieri ${ }^{1}$, Kuwabara ${ }^{5}$ or Suichi ${ }^{5}$ whenever possible.

As studies about POEMS syndrome in SEA were expected to be sparse, case reports, case series, case controls, retrospective and prospective cohorts and non-randomized trials were included. Published peer reviewed journals were given priority although unpublished, partially published and studies published in "gray" literatures were also given consideration to decrease publication bias. English written journals and English-translated journals were prioritized. Since correspondence letters are similar to case reports, they were included.

\subsection{Data Collection}

Both review authors obtained data from eligible studies using a pre-conceptualized data collection form independently. Information collated include country where the journal was published, year, author, type of publication, nationality of the patient, age, sex, chief complaint, reason for initial consult, duration of the disease, initial diagnosis and the presence of classic signs and symptoms of POEMS syndrome. Laboratory and diagnostic examinations such as NCS, serum immunofixation, serum electrophoresis, bone marrow biopsy, CSF analysis, abdominal or chest CT scan, lymph node biopsy, ultrasound, full blood count, liver, and renal function tests and endocrinopathy panels were also extracted. Likewise, the treatment strategy and outcomes were also extracted. In addition, the journals were assessed if they satisfied the mandatory, major and minor criteria set by Dispenzieri. ${ }^{1}$

Due to the heterogeneity and lack of consensus for the application of risk assessment tools for observational studies, assessment of bias was not done. Nevertheless, observational studies were considered as biased and subject to the effect of confounders. Data were analyzed using Microsoft excel.

\section{Results}

\subsection{Result of the Search}

In total, 46 articles were obtained from pubmed, medline and no records were extracted from searching through references. After removing duplicates, the abstracts and titles of 24 articles were reviewed for relevance. Only 13 articles were assessed for eligibility by obtaining the full journal. Of these, 6 were excluded because either the full articles were unobtainable or they did not meet the inclusion criteria (Table 2). Authors were sent electronic mails to request for the full articles to no avail. Only 7 case reports, correspondence and case series were deemed eligible for qualitative synthesis. (Figure 1).

\subsection{Excluded Articles}


Of the 13 articles deemed for analysis, 6 were excluded: 1 review article and 5 case reports. Of the 5 case reports, 2 were not included because they discussed cases of British and Chinese nationalities and 3 had no published full-length articles.

\subsection{Characteristics of Included Studies}

The seven studies spanned from 2007-2020 and included case series of 2 Malaysians ${ }^{14}$; case reports of 1 Indonesian ${ }^{15}, 2$ Malaysians ${ }^{16,17}, 1$ Thai $^{18}$ and 1 Singaporean $^{19}$ and a correspondence of 1 Singaporean ${ }^{20}$ patient. All were written in English. (Table 1)

\subsubsection{Clinical Signs and Symptoms}

The median age of onset of symptom was 54 years (Range: 32-81 years) and the M:F ratio was 1:1. Ordinally, the most common initial presentation was weakness $(4 / 6)$, followed by sudden visual loss $(1 / 6)$ and appearance of hemangioma (1/6). The median time to arriving at POEMS syndrome diagnosis was 5.5 months (range: 2-24 months), with $50 \%$ presenting first as CIDP (2/4). Other associated symptoms include ascites (1/5), weight loss (3/5) and erectile dysfunction and loss of libido $(1 / 5)$.

On physical examination, $67 \%$ had organomegaly (4/6). Ordinally, 2 had hepatosplenomegaly, 1 had splenomegaly and lymphadenopathy and 1 had hepatosplenomegaly and lymphadenopathy. Eighty six percent (6/7) had evidence of hyperpigmentation, hypertrichosis, hemangioma, acrocyanosis and skin nodules while $75 \%$ (3/4) had findings of ascites, peripheral edema and generalized anasarca. Eighty percent (4/5) had papilledema, while all patients had evidence of length dependent polyneuropathy mostly characterized by distal weakness (4/4) and reduced DTR (4/4). Other important signs include clubbing (1), cachexia (1) and the presence of steppage gait (1).

\subsubsection{Laboratory and Diagnostic Examinations}

All patients had abnormal NCS-EMG $(n=8), 7$ demyelinating polyneuropathy with axonal loss and one with pure axonopathy. All but 1 of the 8 patients who tested for serum electrophoresis or immunofixation had presence of monoclonal gammopathy restricted to lambda. Only two patients had VEGF results, of these, only one had elevated level. Only two had abnormal bone marrow biopsy (mean plasma cell \%:5.5, $\mathrm{n}=6$ ). One patient underwent a lymph node biopsy, which turned out to be normal.

Four patients had abnormal CT scan findings $(n=4) ; 2$ had osteosclerotic lesions, 2 had hepatomegaly, and 1 had ascites and pleural effusion. Elevated hemoglobin (4/5), white blood cell (1/5) and platelets $(2 / 5)$ were also recorded. Only one presented with abnormal glucose, TSH and testosterone.

\subsubsection{Treatment and Outcomes}

Five patients were treated with Melphalan and steroid combination while 2 were given immunomodulators ( 1 thalidomide and 1 linalidomide). Only 1 underwent autologous stem cell 
transplantation and 1 was subjected to radiotherapy. All patients improved clinically except for the one who underwent radiotherapy. He died of sepsis.

\subsubsection{Criteria of POEMS Syndrome}

While all papers claimed that POEMS syndrome was present in their cases, only 6 patients satisfied the criteria set by Dispenzieri ${ }^{1}$.(Table 3 ) Whereas all 8 patients fulfilled the minor criteria, only seven patients fulfilled the 2 mandatory criteria and only 6 passed the major criteria. Automatically, if the new proposed criteria by Suichi ${ }^{5}$ was utilized, only 1 will have definitive POEMS syndrome since only 1 had elevated VEGF. Most did not do VEGF testing (6/8).

Of the mandatory criteria, 1 patient did not have monoclonal gammopathy restricted to lambda. Of the major criteria, only 6 patients had findings of sclerotic bone lesions and 1 with elevated VEGF level. None had Castleman Disease. Skin changes was the most common minor criteria (6/7), followed by papilledema (4/6), effusion and edema (5/8), organomegaly (5/8), polycythemia (4/8) and endocrinopathy (3/8). Two mandatory, 1 major and 1 minor Dispenzieri criteria should be fulfilled in order to attain definitive POEMS syndrome diagnosis.

\section{Discussion}

Compared to China, USA and Japan, which reported $1946^{7}, 99^{1}$ and $392^{10}$ POEMS syndrome cases respectively, the combined number of published reports in Southeast Asia was markedly low $(n=7)$. Nevertheless, save for Japan, the true prevalence of the condition is still unknown.

There are several factors that may contribute to the disparity in the number of cases between countries. The difference in genetic make-up and geographic location may explain why only eight cases were reported locally and 1946 cases in the Chinese cohort. ${ }^{7}$ Although there are no studies to support this, conditions such as multiple sclerosis ${ }^{12}$ and $X$-linked dystonia parkinsonism (XDP) ${ }^{13}$ are known to be affected by geography and race respectively. The increased awareness and abundance of diagnostic equipment and specialists in developed countries may also be a factor. Of importance, while there is a move in Japan to include elevated VEGF as one of the mandatory criteria ${ }^{5}$, only 2 cases in our report tested for serum VEGF. Including this criterion as mandatory may further lead to underestimation of the true number of cases in most countries in SEA. The volume of research published in developed countries may cause publication bias. ${ }^{21}$ However, Singapore only reported two case reports despite being a developed country. ${ }^{19,20}$ In addition, population dense countries in Southeast Asia like Malaysia and Philippines, send samples and patients to Singapore for diagnosis of difficult cases.

\subsection{Clinical Manifestations}

Unlike other countries which reported male predominance, the ratio of male and female cases was equal in our study. The median age of 54 was comparable to that of Japan $(54)^{10}$ but higher than USA $(45)^{1}$ 
and China $(46)^{7}$. Consistent with the Chinese $(60.4 \%)^{7}$ and Japanese cohorts $(49 \%)^{10}$, the most common initial presentation was peripheral neuropathy $(66.7 \%)$. Of this, $50 \%$ will initially be diagnosed as CIDP (2/4), which was lower compared to $60 \%$ of both Chinese and Japanese studies. The median duration to correct diagnosis was significantly lower (5.5 months vs 12 months) compared to the Japanese study.

All cases had polyneuropathy since it was required in the mandatory criteria for POEMS syndrome by Dispenzieri. ${ }^{8}$ Only $66.7 \%(4 / 6)$ had evidence of organomegaly on physical examination, lower compared to that of Chinese (83\%) and Japanese cohorts (76.0\%) but higher than US POEMS syndrome patients $(50 \%) .{ }^{1,7,10}$ Some forms of skin changes were found in $85.7 \%$ of our patients, comparable to the Japanese (84\%) but higher than the Chinese (77\%) and USA (68\%) studies. ${ }^{1,7,10}$ Nevertheless, hyperpigmentation was the most common abnormality in all the countries. Like Japan (81\%) and China (82\%), edema and effusion were also prevalent in our study (75\%) with peripheral edema as the most common manifestation. Unlike in the USA where papilledema was reported in only $29 \%$, 4 out of 5 of our case reports $(80 \%)$ had this minor criterion.

\subsection{Laboratory and Diagnostics}

Seven out of eight patients(87.5\%) had diffuse demyelinating polyneuropathy with axonal loss consistent with the findings by Sung et al and in China (98.8\%). $., 7,22,23$ While 29 patients were subjected to VEGF test in China and all cases in the Japanese study, only 1 out of the 2 who had test for VEGF levels was elevated. Using the proposed criteria by Suichi ${ }^{5}$, the one with normal VEGF and the 6 who had never done the test would not qualify as definite POEMS syndrome and will less likely be reported in the literature. Hence the new criteria is only applicable to developed countries. In a national survey of POEMS syndrome in Japan conducted in $2019,84 \%$ had elevated VEGF levels ${ }^{5}$, nevertheless, in a validation study of a new criteria for POEMS syndrome, all the patients included showed elevated VEGF ${ }^{10}$.

All cases in the US, $89 \%$ and $64 \%$ in Japanese and Chinese study respectively had monoclonal gammopathy restricted to lambda. ${ }^{1,7,10}$ In our cohort, 7 out of 8 who had test for monoclonal gammopathy were positive. Most had monoclonal IgG restricted to lambda (6 out of 7) which was higher compared to US (49.4\%). No Castleman syndrome was reported in our study. POEMS syndrome with Castleman Disease was proposed to be removed from the major criteria of Dispenzieri by Misawa et al as this entity had different prognosis compared to the typical POEMS syndrome. ${ }^{3}$

Although lower, the proportion of patients with sclerotic or mixed sclerotic-lytic bone lesions was comparable to US cohort ( $87.5 \%$ vs $97 \%) .{ }^{1}$ Of these, only $50 \%$ were detected using CT scan while the rest were seen using other means. Other findings in the CT scan include organomegaly (2 cases) and effusion (1 case).

Around $73 \%$ and $67 \%$ from the Chinese and US cohorts respectively had endocrinologic abnormality, considerably higher than our $37.5 \%$ proportion. Almost $50 \%$ of our patients presented with polycythemia, erythrocytosis and thrombocytosis, slightly higher than the Japanese study (38\%). 


\subsection{Treatment Options and Outcomes}

Most cases used combination of Melphalan and steroid for treatment (5 out of 8). In contrast, Japanese doctors treat their patients mostly with thalidomide (54\%) and autologous stem cell transplantation $(44 \%)^{10,24}$ Most reports in China used steroids to treat their cases (39.4\%) probably because studies were done prior to the development of current treatment and management. ${ }^{7}$ In the US, management depends on the number of sclerotic lesions. ${ }^{1}$ Only one died of the case reported died while the rest improved clinically. While the prognosis of POEMS syndrome was poor pre-high dose chemotherapy and immunomodulator era, this has significantly improved over the years. ${ }^{3}$

\section{Limitations}

The few number of cases may be an underestimate of the true prevalence hence the study may not be generalizable to the population in Southeast Asia. However, as there are no clinical trials or large observational studies conducted in the region, this study may be the best evidence on the characteristics of the disease in people with Malay descent. The quality of this paper is also dependent on the case reports included. Although the search was comprehensive, we still may have missed some quality papers written in other language considering Southeast Asia consists of countries with diverse cultures and languages. Nevertheless, most Neurology and Hematology societies as well as local journals in Southeast Asia converse and write in English, hence if a paper about POEMS syndrome of good quality is to be published, it will most likely be submitted into an English based local or international journal. Lastly, some diagnostic work up done to establish the diagnosis of POEMS syndrome are incomplete, unavailable, or reported differently. This will cause differences in interpretation depending on the criteria used (i.e., the use of VEGF)

\section{Conclusions}

There were only 8 reported cases of POEMS syndrome in SEA, with the onset of symptoms among middle aged group, with equal sex preponderance and majority with length dependent demyelinating polyneuropathy with abnormal electrodiagnostic studies, monoclonal gammopathy restricted to lambda, papilledema, skin changes, edema, and effusion and organomegaly. Treatment was primarily melphalan and steroid combination. These findings were similar with that of other cohorts in other countries. However due to limitations in the diagnostic tests, recognition, reporting and differences in the criteria used, the true prevalence of the disease in SEA is yet to be determined.

\section{Abbreviations}

POEMS: Polyneuropathy, Organomegaly, Endocrinopathy, M- Protein, Skin Changes 
VEGF: vascular endothelial growth factor

PEP: plasma cell dyscrasia, endocrinopathy, polyneuropathy

CIDP: chronic inflammatory demyelinating polyneuropath

XDP: X-linked dystonia parkinsonism

\section{Declarations}

Originality of submitted research: The content is not published or under review at any other publication.

Funding: None.

Conflicts of interest: None

Ethics approval: Since this is a systematic review, ethics approval was waived.

Consent to participate: Not applicable.

\section{Consent for publication}

All authors have read and agreed the contents of the paper (on initial submission and on any revisions or subsequent resubmissions). Likewise, all authors have agreed to submit this paper on SNCM journal.

Availability of data and material: Not applicable.

Code availability: Not applicable.

\section{Author's contribution:}

MP designed the study. MP and KA collected the studies. MP and KA analyzed the data and wrote the manuscript.

\section{ACKNOWLEDGMENT}

Dr. Prado receives scholarship grant from Takeda Science Foundation.

\section{References}

1. Dispenzieri A, Kyle RA, Lacy MQ, et al. POEMS syndrome: Definitions and long-term outcome. Blood. 2003;101(7):2496-506. doi:10.1182/blood-2002-07-2299.

2. Brown R, Ginsberg L. POEMS syndrome: clinical update. J Neurol. 2019;266(1):268-77. doi:10.1007/s00415-018-9110-6. 
3. Misawa S, Kuwabara S. Polyneuropathy, organomegaly, endocrinopathy, monoclonal gammopathy and skin changes (Crow-Fukase) syndrome: Diagnostic criteria and treatment perspectives. Clin Exp Neuroimmunol. 2013;4(3):318-25. doi:10.1111/cen3.12052.

4. Kuwabara S, Dispenzieri A, Arimura K, Misawa S. Treatment for POEMS (polyneuropathy, organomegaly, endocrinopathy, M-protein, and skin changes) syndrome. Cochrane Database Syst Rev. 2008;(4). doi:10.1002/14651858.CD006828.pub2.

5. Suichi T, Misawa S, Sato Y, et al. Proposal of new clinical diagnostic criteria for POEMS syndrome. J Neurol Neurosurg Psychiatry. 2019;90(2):133-7. doi:10.1136/jnnp-2018-318514.

6. Nasu S, Misawa S, Sekiguchi Y, et al. Different neurological and physiological profiles in POEMS syndrome and chronic inflammatory demyelinating polyneuropathy. J Neurol Neurosurg Psychiatry. 2012;83(5):476-9. doi:10.1136/jnnp-2011-301706.

7. Wang Y, Huang LB, Shi YH, et al. Characteristics of 1946 cases of POEMS syndrome in Chinese subjects: A literature-based study. Front Immunol. 2019;10(JUN):1-12. doi:10.3389/fimmu.2019.01428.

8. Dispenzieri A, Buadi FK. A review of POEMS syndrome. Oncol (United States). 2013;27(12).

9. Bardwick PA, Zvaifler NJ, Gill GN, Newman D, Greenway GD, Resnick DL. Plasma Cell Dyscrasia with Polyneuropathy, Organomegaly, Endocrinopathy, M Protein, and Skin Changes. Med (Baltim). 1980;59(4):311-22. doi:10.1097/00005792-198007000-00006.

10. Suichi T, Misawa S, Beppu M, et al. Prevalence, clinical profiles, and prognosis of POEMS syndrome in Japanese nationwide survey. Neurology. 2019;93(10):E975-83. doi:10.1212/WNL.0000000000008062.

11. Soubrier MJ, Dubost JJ, Sauvezie BJM. POEMS syndrome: A study of 25 cases and a review of the literature. Am J Med. 1994;97(6):543-53. doi:10.1016/0002-9343(94)90350-6.

12. Wade BJ. Spatial Analysis of Global Prevalence of Multiple Sclerosis Suggests Need for an Updated Prevalence Scale. Mult Scler Int. 2014;2014:1-7. doi:10.1155/2014/124578.

13. Evidente VGH, Lyons MK, Wheeler M, et al. First case of X-linked dystonia-parkinsonism ("Lubag") to demonstrate a response to bilateral pallidal stimulation. Mov Disord. 2007;22(12):1790-3. doi:10.1002/mds.21420.

14. Lau YH, Mohd Unit H, Lee LP, Loh WK, Hiew FL. Temporal dispersion in demyelination of POEMS syndrome and Castleman disease. Clin Neurophysiol Pract. 2020;5:112-7. doi:10.1016/j.cnp.2020.05.001.

15. Oehadian A, Prasetya D, Fadjari TH. POEMS syndrome: a rare case of monoclonal plasmaproliferative disorder. Acta Med Indones. 2010;42(2):100-3.

16. Low JM, Binti Basiam S, Binti Kori AN. POEMS syndrome: A rare paraneoplastic presentation of spinal plasmacytoma. Med J Malaysia. 2019;74(4):335-7.

17. Gao XM, Chang JM. POEMS syndrome: A case report. J Clin Dermatology. 2010;39(11):708-10. 
18. Jindahra P, Dejthevaporn C, Niparuck P, et al. Atypical central retinal artery occlusion as the first presentation of POEMS syndrome: A case report. BMC Neurol. 2018;18(1):4-10. doi:10.1186/s12883-018-1071-y.

19. Rathakrishnan R, Liu TC, Chan YC, Ong BKC. POEMS syndrome - A case for more aggressive treatment. Ann Acad Med Singapore. 2007;36(6):435-7.

20. Chen XF, Ong NWR, Tang PY, Pang SM, Sittampalam K. Glomeruloid haemangioma pattern in reactive angioendotheliomatosis leading to the diagnosis of POEMS syndrome. Pathology. 2020; (xxx):10-12. doi:10.1016/j.pathol.2020.07.016.

21. Jaffe K, ter Horst E, Gunn LH, Zambrano JD, Molina G. A network analysis of research productivity by country, discipline, and wealth. PLoS One. 2020;15(5):1-15. doi:10.1371/journal.pone.0232458.

22. Sung JY, Kuwabara S, Ogawara K, Kanai K, Hattori T. Patterns of nerve conduction abnormalities in poems syndrome. Muscle Nerve. 2002;26(2):189-93. doi:10.1002/mus.10182.

23. Kamil K, Yazid MD, Idrus RBH, Das S, Kumar J. Peripheral Demyelinating Diseases: From Biology to Translational Medicine. Front Neurol. 2019;10(March):1-12. doi:10.3389/fneur.2019.00087.

24. Misawa S, Sato $Y$, Katayama K, et al. Safety and efficacy of thalidomide in patients with POEMS syndrome: a multicentre, randomised, double-blind, placebo-controlled trial. Lancet Neurol. 2016;15(11):1129-37. doi:10.1016/S1474-4422(16)30157-0.

25. Kasinathan G, Sathar J. Ascites as a presenting sign of multicentric mixed-type Castleman disease variant of POEMS syndrome. Hematol Transfus Cell Ther. 2020; $(x \mathrm{x}): 7-10$. doi:10.1016/j.htct.2020.01.007.

\section{Tables}

Due to technical limitations, table 1 is only available as a download in the Supplemental Files section.

Table 2: Table of excluded studies

\begin{tabular}{|llll|}
\hline Author & Year & Country & Reason for Exclusion \\
\hline Kamil $^{23}$ & 2019 & Malaysia & Review article \\
\hline Kasinathan $^{25}$ & 2020 & Malaysia & Chinese patient \\
\hline Intragumtornchai & 1993 & Thailand & No full article \\
\hline Limvorapitak & 2017 & Thailand & British patient \\
\hline Witoonpanich & 2005 & Thailand & No full article \\
\hline Witoonpanich & 1988 & Thailand & No full article \\
\hline
\end{tabular}

Table 3: Proportion of fulfilled POEMS Syndrome Criteria by Dispenzieri 


\begin{tabular}{|c|c|c|}
\hline Criteria & $\begin{array}{l}\text { No. of Patients Satisfying the } \\
\text { Criteria }\end{array}$ & $\%$ \\
\hline Polyneuropathy ${ }^{a}(n=8)$ & 8 & 100 \\
\hline $\begin{array}{l}\text { Monoclonal gammopathy restricted to } \lg A \text { or } \lg G \\
(\text { lambda })^{a}(n=8)\end{array}$ & 7 & 87.5 \\
\hline Sclerotic bone lesions ${ }^{b}(n=8)$ & 6 & 85.7 \\
\hline Elevated VEGFb $(n=2)$ & 1 & 50.0 \\
\hline Castleman Disease $^{\mathrm{b}}$ & 0 & 0 \\
\hline Skin Changes $^{\mathrm{C}}(\mathrm{n}=7)$ & 6 & 85.7 \\
\hline Papilledema ${ }^{c}(n=6)$ & 4 & 66.7 \\
\hline Effusion and Edema ${ }^{c}(n=8)$ & 5 & 62.5 \\
\hline Organomegaly $^{c}(n=8)$ & 5 & 62.5 \\
\hline $\begin{array}{l}\text { Polycythemia/Erythrocytosis/ } \\
\text { Thrombocytosis }{ }^{\mathrm{C}}(\mathrm{n}=8)\end{array}$ & 4 & 50.0 \\
\hline Endocrinopathy ${ }^{\mathrm{c}}(\mathrm{n}=8)$ & 3 & 37.5 \\
\hline
\end{tabular}

${ }^{\mathrm{a}}$ Mandatory, ${ }^{\mathrm{b}}$ Major, ${ }^{\mathrm{c}}$ Minor

\section{Supplemental Material}

Supplemental Material is not available with this version

\section{Figures}




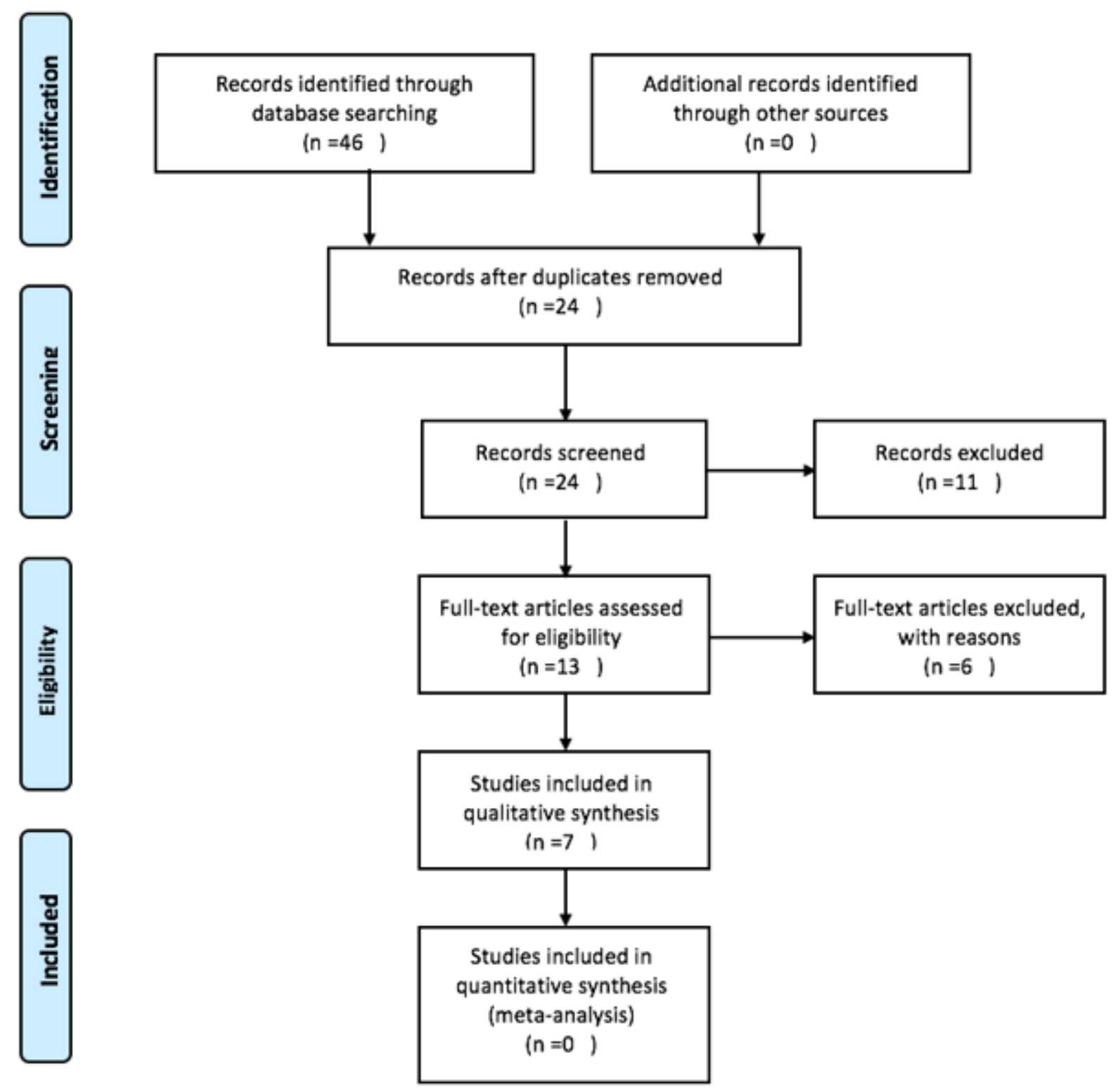

Figure 1

The PRISMA flow diagram

\section{Supplementary Files}

This is a list of supplementary files associated with this preprint. Click to download.

- Table1.docx 